\title{
Ruggedness and reproducibility of the MBEC biofilm disinfectant efficacy test
}

\section{Authors: Albert E. Parker, Diane K. Walkera, Darla M. Goeres, N. Allan, M.E. Olson, \& A. Omar}

NOTICE: this is the author's version of a work that was accepted for publication in Journal of Microbiological Methods. Changes resulting from the publishing process, such as peer review, editing, corrections, structural formatting, and other quality control mechanisms may not be reflected in this document. Changes may have been made to this work since it was submitted for publication. A definitive version was subsequently published in Journal of Microbiological Methods, 102, July 2014, DO\#10.1016/j.mimet.2014.04.013.

Parker AE, Walker DK, Goeres DM, Allan N, Olson ME, Omar A, "Ruggedness and reproducibility of the MBEC biofilm disinfectant efficacy test," Journal of Microbiological Methods, July 2014 102:55-64 


\title{
Ruggedness and reproducibility of the MBEC biofilm disinfectant efficacy test
}

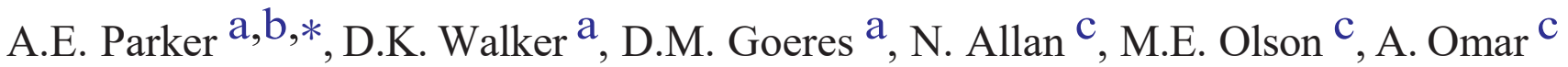 \\ a Center for Biofilm Engineering, Montana State University, Bozeman, MT 59715, USA \\ b Department of Mathematical Sciences, Montana State University, Bozeman, MT 59715, USA \\ c Innovotech Inc., Edmonton AB T6N 1H1, Canada
}

A B S T R A C T

The MBECTM Physiology \& Genetics Assay recently became the first approved ASTM standardized biofilm disinfec-tant efficacy test method. This report summarizes the results of the standardization process using Pseudomonas aeruginosa biofilms. Initial ruggedness testing of the MBEC method suggests that the assay is rugged(i.e., insensitive) to small changes to the protocol with respect to 4 factors: incubation time of the bacteria (when varied from 16 to $18 \mathrm{~h}$ ), treatment temperature $\left(20-24{ }^{\circ} \mathrm{C}\right)$, sonication duration (25-35 $\mathrm{min})$, and sonication power (130-480 W). In order to assess the repeatability of MBEC results across multiple tests in the same laboratory and the reproducibility across multiple labs, an 8-lab study was conducted in which 8 concentrations of each of 3 disinfectants (a non-chlorine oxidizer, a phenolic, and a quaternary ammonium compound) were applied to biofilms using the MBEC method. The repeatability and reproducibility of the untreated control biofilms were acceptable, as indicated by small repeatability and reproducibility standard deviations (SD) (0.33 and $0.67 \log 10\left(\mathrm{CFU} / \mathrm{mm}^{2}\right)$, respectively). The repeatability SDs of the biofilm log reductions after application of the 24 concentration and disinfectant combinations ranged from 0.22 to 1.61 , and the reproducibility SDs ranged from 0.27 to 1.70 . In addition, for each of the 3 disinfectant types considered, the assay was statistically signifi-cantly responsive to the increasing treatment concentrations.

\section{N T R O D U C T I O N}

Members of academia, industry and regulatory agencies have come to recognize that disinfectant efficacy against biofilms (i.e. microorgan-isms attached to surfaces) cannot be adequately studied by traditional microbiological methods that were developed for planktonic cells (e.g., see Donlan and Costerton, 2002; Davies, 2003; Fux et al., 2004; Buckingham-Meyer et al., 2007; Behnke et al., 2011). Thus, suspension tests and dried surface tests (currently used to support hospital disinfectant claims in the US (Tomasino et al., 2012)) are not appropriate to test disinfectant efficacy against biofilms. Although regulatory agencies in the US still have not adopted any method to substantiate biofilm efficacy claims, this is expected in the next few years.

Biofilm methods for research purposes have been available for decades. Only in the past few years, however, have some of these research methods completed the arduous process to become available 
concentration (MBEC) device, a low shear reactor system where the biofilm is grown under batch conditions (i.e., there is no continuous supply of media to the biofilm), complements this suite of available biofilm growth methods.

The MBEC device (originally referred to as the "Calgary device"), depicted in Fig. 1, is a widely used, 96-well plate system for growing biofilm that allows for high throughput screening (Ceri et al., 1999; Harrison et al., 2010). Biofilm is grown on pegs on a lid that fits over the 96 wells. With such a large number of wells, the pegs with the established biofilm may be immersed in multiple treatments and controls simultaneously. The layout of the 96 wells used in the tests described in this paper is depicted in Fig. 2. Forty wells were used to study the effect of the application of 8 concentrations of disinfectant on the biofilm bacteria, while the remaining wells contained various media or reagents as controls.

The MBEC assay was the first protocol approved by ASTM that is both a biofilm growth and disinfectant protocol (ASTM E2799). It is also the first ASTM biofilm method substantiated by a multilaboratory (collaborative) study (ASTM RR:E35-1006, 2011). The purpose of the collaborative study was to quantitatively assess: the repeatability across multiple tests and the reproducibility across multiple labs of the biofilm log reductions after application of the disinfectant treatments; the resemblance of the control biofilms across labs; and the responsiveness of the assay to detect increased log reductions in the biofilm as the concentration of the chemical was increased.

Before the collaborative study began, the MBEC assay was subjected to a ruggedness test in order to assess the sensitivity of the assay to small changes of four important operational factors. Ruggedness test results provide regression equations that quantify how changes in the operational factors affect the responses of the MBEC assay. The magnitudes of these changes were chosen to reflect the range of factor values that the method may be subjected to by multiple users at different labs. Ruggedness testing is rarely used in microbiology, which is unfortunate as there are many benefits of this predictive test. Two standardized biofilm growth methods have previously been subjected to ruggedness testing: the CDC reactor (Goeres et al., 2005) and the DFR (Goeres et al., 2009).

One purpose of this paper is to report the performance of the MBEC assay in the ruggedness test and in the subsequent collaborative study. We predict that this is the first of an infusion of new standardized methods needed to assess anti-biofilm efficacy over the next decade. Thus, the second purpose of this paper is to help inform this future work by clearly elucidating the steps that we took to standardize the first disinfectant test against biofilms.

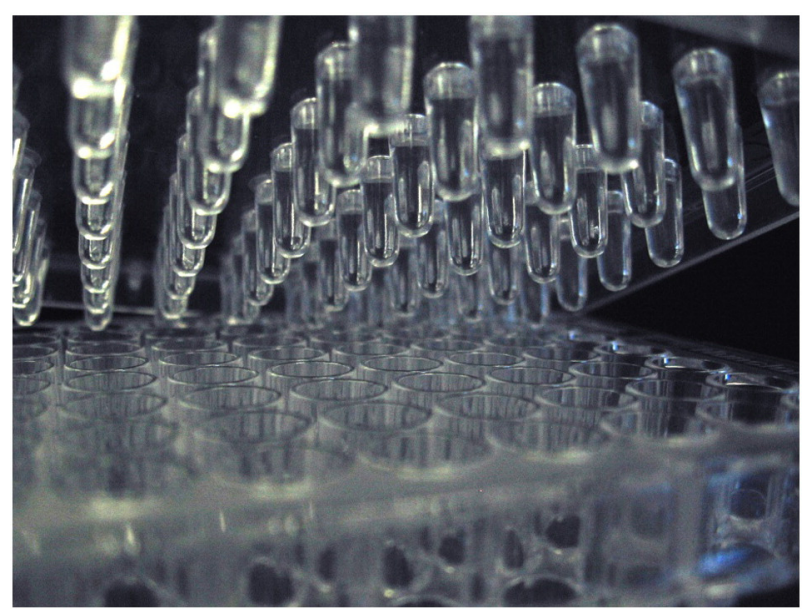

Fig. 1. A photograph of the MBEC plate.

\section{Methods}

\subsection{The MBEC method}

\subsubsection{Growth and treatment}

To initiate the standardized MBEC method (ASTM E2799), the wells of an MBEC device (Innovotech Inc., Edmonton, Ab. Canada), excluding those for sterility control (Fig. 2, "SC"), were filled with $150 \mu \mathrm{L}$ of tryptic soy broth inoculated with $10^{5}$ cells/mL of $P$. aeruginosa ATCC 15442 , and then incubated for $16-18 \mathrm{~h}$ at $35 \pm 2{ }^{\circ} \mathrm{C}$ on an orbital shaker at $110 \pm 10 \mathrm{rpm}$ to establish a biofilm on the pegs located on the lid of the device. Once the biofilm was grown, 5 pegs were detached from the lid to determine the initial density of bacteria (Fig. 2, "PC") by immersing each peg into $0.2 \mathrm{~mL}$ buffered water, and then using sonication to remove the biofilm from the pegs and to disaggregate the biofilm into the $0.2 \mathrm{~mL}$ volume. A $10 \mu \mathrm{L}$ volume was removed from this $0.2 \mathrm{~mL}$ volume for a 10 -fold dilution series. A "challenge plate" was prepared according to the layout in Fig. 2 with each well containing $200 \mu \mathrm{L} /$ well of treatment(s) or control media/reagents. The lid with the remaining biofilm-covered pegs was placed on top of the challenge plate for the specified contact time for the disinfectant treatment. The lid was then transferred to a 96 well plate with each well containing $200 \mu \mathrm{L}$ of neutralizer, and then the biofilm on the remaining 91 pegs was sonicated for $30 \mathrm{~min}$ to remove and disaggregate the biofilm. The contents of each row were then transferred to a new 96 well plate for serial dilution followed by spot plating $10 \mu \mathrm{L}$ on tryptic soy agar (TSA) in order to determine viable cell concentrations.

\subsubsection{Device layout}

As depicted in Fig. 2, for each treatment, the first 5 wells of row A contained the highest concentration of disinfectant; row $B$ contained half the concentration (50\%) compared to row A; row $C$ contained half the concentration from row $B(25 \%)$; and so on until the most dilute concentration in row $\mathrm{H}$ contained half the concentration from row $\mathrm{G}(0.78 \%)$. Column 6 contained a volume of the disinfectant at the $100 \%$ concentration mixed with an equal volume of neutralizer ("50:N"). The wells of column 7 contained neutralizer only ("N"). Column 8 contained buffered water and was the untreated control ("UC"). Columns 9-11 are not used in the standard method and remain empty as do the wells in rows D-H of column 12 since the pegs are used for preliminary biofilm counts ("PC"). Rows A-C of column 12 contained sterility (negative) controls ("SC") of the growth media, buffered water, and neutralizer.

\subsection{Quantification of biofilm bacteria}

At each dilution $d$ in the serial dilution series, the number of colony forming units (CFU) was enumerated, scaled up by the dilution factor $10^{d}$ and then reported as $10^{d} \times \mathrm{CFU} / 10 \mu \mathrm{L}$. These bacterial counts were then converted to a log density (LD) per $\mathrm{mm}^{2}$ of surface area using the following equation,

$$
\mathrm{LD}=\log _{10}\left(\mathrm{CFU} / \mathrm{mm}^{2}\right)=\log _{10}\left[\left(10^{d} \mathrm{CFU} / 10 \mu \mathrm{L}\right)(V / S A)\right]
$$

where $V$ is the volume sonicated into $(0.2 \mathrm{~mL})$; and $S A$ is the surface area of the peg $\left(46.63 \mathrm{~mm}^{2}\right)$. Whenever no CFUs were observed in all dilutions, a $1 \frac{1}{2}$ CFU was substituted in at the lowest dilution plated, and then scaled up (Hamilton et al., 2013). Since the 0th dilution was always plated, then after applying the CFU substitution rule,

$$
\begin{aligned}
\mathrm{LD} & =\log _{10}\left[(0.5 \mathrm{CFU} / 0.01 \mathrm{~mL})\left(0.2 \mathrm{~mL} / 46.63 \mathrm{~mm}^{2}\right)\right] \\
& =\log _{10}\left[0.2145 \mathrm{CFU} / \mathrm{mm}^{2}\right]=-0.67
\end{aligned}
$$

was the substituted value for the LD. Note that changing units to $\log _{10}$ $\left(\mathrm{CFU} / \mathrm{cm}^{2}\right)$ would increase all LDs by +2 , and leave the standard 


\begin{tabular}{|c|c|c|c|c|c|c|c|c|c|c|c|c|}
\hline & 1 & 2 & 3 & 4 & 5 & 6 & 7 & 8 & 9 & 10 & 11 & 12 \\
\hline A & 100 & 100 & 100 & 100 & 100 & $50: \mathrm{N}$ & $\mathrm{N}$ & UC & & & SC \\
\hline B & 50 & 50 & 50 & 50 & 50 & $50: \mathrm{N}$ & N & UC & & & SC \\
\hline C & 25 & 25 & 25 & 25 & 25 & $50: \mathrm{N}$ & $\mathrm{N}$ & UC & & & SC \\
\hline D & 12.5 & 12.5 & 12.5 & 12.5 & 12.5 & $50: \mathrm{N}$ & $\mathrm{N}$ & UC & & & PC \\
\hline E & 6.25 & 6.25 & 6.25 & 6.25 & 6.25 & $50: \mathrm{N}$ & $\mathrm{N}$ & UC & & & PC \\
\hline F & 3.125 & 3.125 & 3.125 & 3.125 & 3.125 & $50: \mathrm{N}$ & $\mathrm{N}$ & UC & & & PC \\
\hline G & 1.5625 & 1.5625 & 1.5625 & 1.5625 & 1.5625 & $50: \mathrm{N}$ & $\mathrm{N}$ & UC & & & PC \\
\hline H & 0.7813 & 0.7813 & 0.7813 & 0.7813 & 0.7813 & $50: \mathrm{N}$ & $\mathrm{N}$ & UC & & & PC \\
\hline
\end{tabular}

Fig. 2. Layout of the MBEC plate used in the ruggedness testing and multi-lab study. Section 2.1.2 describes the acronyms (50:N, N, UC, SC) used in the figure.

deviation (SD) of the LDs unchanged. In other words, the SD of the LDs is unitless.

Two separate microbial responses were considered in all subsequent statistical analyses. First, the mean LD of organisms attached to the 8 untreated control pegs (in column 8, labeled "UC") of each MBEC plate was calculated. Second, on each plate, the response of the biofilm grown on pegs and then subjected to disinfectants at the 8 different concentrations was quantified by the log reduction (LR), which is the mean LD of biofilm on the eight untreated control pegs minus the mean LD of biofilm that survived on the five pegs treated with a disinfectant in one of rows A-H in columns 1-5. Thus, the LR is unitless. For each treatment concentration on a plate, denoted by rows $A, B, C$, $\mathrm{D}, \mathrm{E}, \mathrm{F}, \mathrm{G}$, and $\mathrm{H}$ in the MBEC plate, one LR is calculated.

\subsection{Neutralization}

For all disinfectants and concentrations considered in this paper, $\mathrm{D} / \mathrm{E}$ neutralizing broth was used (Neogen Corporation, Lansing, MI). The MBEC challenge plate contained two columns, each with 8 wells that allow for the evaluation of the neutralizer in two ways (Fig. 2): (1) was the disinfectant neutralized? and (2) does the neutralizer itself impact the biofilm? To answer these questions, the eight wells in column 6, labeled "50:N", contain a neutralized disinfectant: $100 \mu \mathrm{L}$ of the $100 \%$ concentration of the disinfectant and $100 \mu \mathrm{L}$ of the neutralizer. The biofilms grown on pegs and then subjected to these wells were compared to the controls to determine whether the neutralizer satisfactorily neutralized the disinfectant. This 1:1 disinfectant to neutralizer ratio in the " $50: \mathrm{N}$ " column is a conservative check of the neutralizer's ability to inactivate the active compound. In practice, the biofilmladen lid that was exposed to the disinfectant is transferred to a fresh microtiter plate containing only neutralizer, in which case the disinfectant to neutralizer ratio is much larger (about 1:200 to 3:200, cf. Section 2.1.1). The eight wells in column 7, labeled "N," contain just neutralizer. Comparing the biofilm subjected to these wells to the untreated control biofilms determined whether the neutralizer itself inhibits or otherwise affects biofilm bacteria. A mixed effects statistical model was used to test the efficacy of the neutralizer for each disinfectant separately, with nested random effects due to lab, experimental day within a lab, and multiple wells within a plate per day. Treatment (with three levels, neutralized disinfectant (in column "50:N"), Neutralizer (in column "N"), and the controls (in column "UC")) was the fixed effect. As is commonly done for planktonic assays using suspended bacteria, all pairwise comparisons of the mean LDs amongst these levels were performed (ASTM E1054). These comparisons were made using Tukey's multiple comparison procedure. However, since we believe that it is more informative to assess statistical equivalence as opposed to failing to find statistical differences when assessing neutralizers, equivalence tests were performed by calculating 3 simultaneous 2-sided 90\%
Tukey confidence intervals for the true mean differences. If the $90 \%$ confidence intervals were contained in the interval $[-0.5,0.5]$ (Nelson et al., 2013), then statistical equivalence was concluded at 95\% confidence (Richter and Richter, 2002; ASTM E2935). In other words, we assumed that mean LD differences less than $1 / 2 \log$ were negligible and not of practical importance.

\subsection{Ruggedness testing}

A ruggedness test determines the sensitivity of a protocol to small changes in operational factors. For the ruggedness test of the MBEC assay, the factors tested were treatment temperature (Temp), sonication duration (SonDur), sonication power (Power), and incubation time (Inc). The non-chlorine oxidizer disinfectant Virkon (DuPont, Wilmington, DE) was used in all ruggedness experiments. For each MBEC test, Virkon was applied in 8 different concentrations (Fig. 2), starting at $535 \mathrm{mg} / \mathrm{L}(100 \%)$ in the five wells in row A of the plate, and then with half concentrations applied in each row thereafter (thus, $4.2 \mathrm{mg} / \mathrm{L}$ was tested in row $\mathrm{H}$ ).

\subsubsection{Ruggedness test experimental design}

In order to estimate both main effects and two-way interactions amongst the four factors, experiments were run at low, medium and high levels of the factors of interest (Table 1). These low and high levels were chosen in order to span a realistic range of deviations to the protocol when conducted in different labs. Experiments at specific combinations of the factors were chosen according to a fractional factorial experimental design and the run order was randomized as outlined in Table 2. Two of the ten pairs of experiments (runs 1 and 8) were run at standard operating procedure (SOP) values for all of the factors. These repeats (called "center points" in the statistical literature) allowed preliminary estimation of the variability of the control LDs and LRs when the method was run according to the SOP. Two technicians each performed the ten experiments in Table 2 side-by-side in order to minimize technician-to-technician variability in the experimental outcomes, thereby increasing the statistical power to detect

Table 1

The four factors to be analyzed in the ruggedness testing, the short name used in subsequent tables, and the low, medium and high levels of each factor.

\begin{tabular}{lllll}
\hline Factor & Short name & Low & Medium (SOP) & High \\
\hline Incubation time & Inc & $16 \mathrm{~h}$ & $17 \mathrm{~h}$ & $18 \mathrm{~h}$ \\
Treatment temperature & Temp & $20{ }^{\circ} \mathrm{C}$ & $22{ }^{\circ} \mathrm{C}$ & $24{ }^{\circ} \mathrm{C}$ \\
Sonication power & Power & $130 \mathrm{~W}$ & $250 \mathrm{~W}$ & $480 \mathrm{~W}$ \\
Sonication duration & SonDur & $25 \mathrm{~min}$ & $30 \mathrm{~min}$ & $35 \mathrm{~min}$ \\
\hline
\end{tabular}


Table 2

Fractional factorial schedule of ruggedness tests for one disinfectant and one technician.

\begin{tabular}{lllll}
\hline Run & Inc & Temp & Power & SonDur \\
\hline 1 & $17 \mathrm{~h}$ & $22{ }^{\circ} \mathrm{C}$ & $250 \mathrm{~W}$ & 30 \\
2 & $18 \mathrm{~h}$ & $20{ }^{\circ} \mathrm{C}$ & $130 \mathrm{~W}$ & 25 \\
3 & $16 \mathrm{~h}$ & $24{ }^{\circ} \mathrm{C}$ & $480 \mathrm{~W}$ & 35 \\
4 & $18 \mathrm{~h}$ & $24{ }^{\circ} \mathrm{C}$ & $480 \mathrm{~W}$ & 25 \\
5 & $18 \mathrm{~h}$ & $24{ }^{\circ} \mathrm{C}$ & $130 \mathrm{~W}$ & 35 \\
6 & $18 \mathrm{~h}$ & $20{ }^{\circ} \mathrm{C}$ & $480 \mathrm{~W}$ & 35 \\
7 & $16 \mathrm{~h}$ & $20{ }^{\circ} \mathrm{C}$ & $480 \mathrm{~W}$ & 25 \\
8 & $17 \mathrm{~h}$ & $22{ }^{\circ} \mathrm{C}$ & $250 \mathrm{~W}$ & 30 \\
9 & $16 \mathrm{~h}$ & $20{ }^{\circ} \mathrm{C}$ & $130 \mathrm{~W}$ & 35 \\
10 & $16 \mathrm{~h}$ & $24{ }^{\circ} \mathrm{C}$ & $130 \mathrm{~W}$ & 25 \\
\hline
\end{tabular}

the effects of the four factors. Thus, the ruggedness test results are based on $20 \mathrm{MBEC}$ runs, all performed in the same lab.

\subsubsection{Assessing factor effects}

To assess the effect of each of the four factors of interest, a nested mixed effects statistical model was fit separately to the control LDs and the LRs. The three nested random effects in the model were technician, experiments (or plates) nested within technician, and wells within an experiment. The fixed effects in the models were the four factors given in Tables 1, and 3 of the 6 two-way interactions. A covariate for "Biofilm Growth" was also included, defined as the mean LD of the "PC" pegs in the MBEC plate column 12 (Fig. 2). Interactions were assessed via interaction plots and significance tests.

The statistical model for the LRs was the same as just described except that, instead of a covariate for Biofilm Growth, it included a covariate for disinfectant concentration. From the LRs calculated across the 8 different disinfectant concentrations in rows A-H on the MBEC challenge plate, a dose-response curve was plotted. The covariate for disinfectant concentration (DisConc) simply characterizes this curve with a line. For ease of interpretability, the covariate for disinfectant concentration in MBEC plate rows $\mathrm{A}, \mathrm{B}, \mathrm{C}, \ldots, \mathrm{H}$ was encoded as the whole numbers $7,6,5,4, \ldots, 0$ respectively. These values are equivalent to taking the transform DisConc $=\log _{2}$ (nominal concentration in $\mathrm{mg} / \mathrm{L}$ ) $\log _{2}(4.2 \mathrm{mg} / \mathrm{L})$ where $4.2 \mathrm{mg} / \mathrm{L}$ is the lowest concentration of Virkon used (in row $\mathrm{H}$ of the MBEC challenge plate).

The output of the statistical analyses is a regression equation, with a coefficient estimate and significance test for each factor and two-way interaction. The regression equations always contain all the main effects regardless of statistical significance, but only contain statistically significant two-way interactions. A small coefficient in the regression equation suggests that a response (i.e., either the mean control LD or the LR) is rugged with respect to small changes in the corresponding factor. Caution in interpretation should be taken, however, since each coefficient scales by the units of the factor, i.e., incubation time $(\mathrm{h})$, sonication duration ( $\mathrm{min})$, sonication power $(\mathrm{W})$ and treatment temperature $\left({ }^{\circ} \mathrm{C}\right)$. The effect of each factor was further assessed by significance tests, main effect plots, and interaction plots.

Table 3

Neutralization results from the 8-lab collaborative study. Satisfactory neutralization is concluded if all $90 \%$ confidence intervals $(\mathrm{CI})$ are contained in the equivalence interval of $[-0.5,0.5]$.

\begin{tabular}{llrll}
\hline Disinfectant & Comparison & Mean LR & SE(LR) & $90 \% \mathrm{CI}$ \\
\hline \multirow{2}{*}{ Non-chlorine oxidizer } & Neutralized disinfectant & -0.09 & 0.0405 & {$[-0.17,-0.01]$} \\
& Neutralizer toxicity & -0.04 & 0.0405 & {$[-0.12,0.04]$} \\
Phenol & Neutralized disinfectant & 0.67 & 0.0526 & {$[0.56,0.77]$} \\
\multirow{2}{*}{ Quat } & Neutralizer toxicity & -0.09 & 0.0526 & {$[-0.19,0.02]$} \\
& Neutralized disinfectant & 0.21 & 0.0424 & {$[0.13,0.30]$} \\
& Neutralizer toxicity & -0.06 & 0.0424 & {$[-0.14,0.03]$} \\
\hline
\end{tabular}

\subsection{Collaborative study}

\subsubsection{Study design}

An 8-lab study was conducted in 2011 as the last step of the process towards standardization of the MBEC assay with ASTM. Specific details of this study have been documented as a research report (ASTM RR: E35-1006, 2011). The main goal of this collaborative study was to determine the resemblance of the control LDs and of the repeatability and reproducibility of the LRs generated by the MBEC protocol. The responsiveness of the method to increasing concentrations of each treatment was also assessed (Hamilton et al., 2013).

All 8 laboratories participated in an on-line training session prior to the study. During the collaborative study, 3 days of testing were performed at each lab. On each day, 3 disinfectants were tested, each on a separate plate. This experimental design satisfies ASTM's minimal requirements of 6 labs, 3 products, and 3 repeated tests of each product at each lab (ASTM E691). The 3 disinfectants used in this study were chosen to represent different classes of products: Virkon (DuPont, Wilmington, DE), a non-chlorine oxidizer (also used in the ruggedness test); Multi-Phenolic Disinfectant (Bio Agri Mix LP, Mitchell, Ontario, CA) (a phenol); and Biosentry 904 (Neogen Corporation, Lansing, MI), a quaternary ammonium compound (quat). The ranges of concentrations used in rows A-H of the MBEC plates for each of the 3 disinfectants were $535-4.2 \mathrm{mg} / \mathrm{L}$ for the oxidizer; $1640-13 \mathrm{mg} / \mathrm{L}$ for the phenol; and $3680-29 \mathrm{mg} / \mathrm{L}$ for the quat. Since the goal of the study was to assess the MBEC method and not these products, these products are referred to throughout the manuscript by their generic names.

There were a few missing data, apparently due to the spot plating techniques. One lab reported a missing tip on the low-volume multichannel-pipetter, and another lab reported that when spot plating "sometimes it happened that certain drops came into contact with the direct drop nearby." At Lab 5, the phenol treatment on Day 2 had measurements from only 4 out of 5 pegs; the quat on Day 2 had only 4 measurements; and the quat on Day 3 had only 4 measurements. At Lab 6, there was no row $\mathrm{H}$ data for the quat on Day 1 for either the disinfected or control biofilms.

All questions regarding the data were brought to the attention of the study director. All data were validated, and since no deviations from the study protocol were identified, all data collected from all 8 labs were included in the statistical analyses (consistent practice with the guidelines in section 19 of ASTM E691).

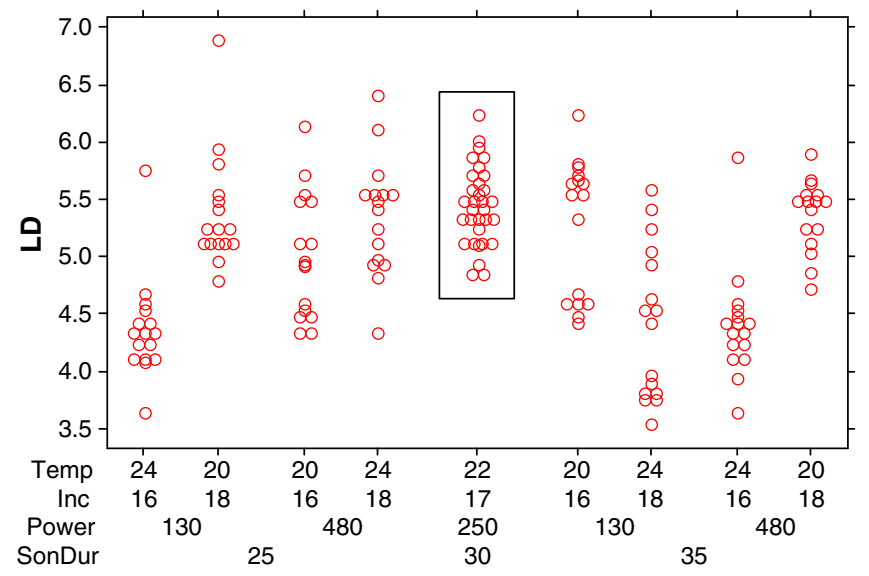

Fig. 3. Pooled across 2 technicians and 20 plates used in the ruggedness tests, the log densities $\left(\mathrm{LD}=\log _{10}\left(\mathrm{CFU} / \mathrm{mm}^{2}\right)\right)$ of the untreated control biofilms grown using the MBEC are plotted. Each point corresponds to the LD of biofilm bacteria on a single control peg. The data in the rectangle are from plates 1 and 8 per technician that were run at the SOP values of the four operational factors. 
Table 4

Summary statistics for the regression equation fit to the log densities (LD $=\log _{10}$ (CFU/ $\mathrm{mm}^{2}$ )) of untreated control biofilms from the ruggedness tests. Units for the factors are provided in Table 1 . Since there were no significant 2 -way interactions, the model contains only main effects.

\begin{tabular}{lclc}
\hline Factors & Estimate & SE & $p$-value \\
\hline Intercept & 5.027 & 0.2445 & $<0.00005$ \\
Inc-17 & 0.1111 & 0.1146 & 0.3500 \\
SonDur-30 & -0.0042 & 0.0224 & 0.8534 \\
Temp-22 & -0.1178 & 0.0572 & 0.0601 \\
Power-250 & 0.00036 & 0.0006 & 0.5616 \\
BiofilmGrowth-5.87 & 0.3893 & 0.2217 & 0.1026 \\
\hline
\end{tabular}

2.5.2. Assessing resemblance, repeatability and reproducibility of the MBEC method

To assess the resemblance of the LDs of untreated control biofilms across labs, a statistical model was fit with nested random effects due to lab, experimental day within a lab, plates within an experimental day, and wells within a plate. It was possible to estimate the plate variability within each day because each lab ran three plates on each test day, one for each of the three disinfectants considered. The controls from all three of these plates were considered simultaneously in the same statistical analysis since they were processed similarly, including the use of the same D/E neutralizer. The model just described gives a pooled estimate of the repeatability SD of the method at a single lab by pooling the repeatability variances across all labs. The repeatability SD was also calculated at each lab separately using a model with random effects for experimental day, plates within an experimental day, and wells within a plate. The repeatability and reproducibility for the LR for each disinfectant and concentration were also assessed using a mixed model with a random effect for lab.

\subsubsection{Assessing responsiveness of the MBEC method}

To assess responsiveness of the method to the 8 increasing disinfectant concentrations in rows A-H in the challenge plate, a dose-response curve with respect to the LR was constructed. One approach to quantify responsiveness is with the slope of a regression line. Such a line was fit to the LRs for each disinfectant using a mixed effects model with random effects due to lab and tests within a lab, and a covariate for disinfectant concentration (see Section 2.4.2).

\subsection{Other statistical details}

The analysis of the collaborative study data followed the guidelines for disinfectant tests described in Hamilton et al. (2013). Mixed effects models (Pinheiro and Bates, 2000) specifically for disinfectant efficacy data are described in detail by Parker and Hamilton (2011). The method of restricted maximum likelihood method was used to fit the nested mixed models using the software R v.2.11.0 (R Core Team, 2012), package nlme (Pinheiro et al., 2012); the R code is provided in Parker and Hamilton (2013). Three types of plots were used to identify outliers and to check the fit of the statistical models: individual value, residual and normal probability plots. An additional check for outliers was to perform the hypothesis tests recommended by ASTM (cf. sections 16 and 17 in ASTM E691). All graphs were rendered by Minitab v.16. Minitab was also used to generate the fractional factorial experimental design for the ruggedness testing. All statistical tests, including the equivalence tests, were conducted at a significance level of $5 \%$.

\section{Results}

\subsection{Neutralization}

The results of the neutralization tests conducted during the collaborative study are presented in Table 3. For all 3 disinfectants tested, the mean LD of biofilm bacteria subjected to the control or neutralizer-only wells were statistically equivalent. For the nonchlorine oxidizer and the quat, the mean LD of biofilm bacteria subjected to the control or neutralized disinfectant wells were also statistically equivalent. Unfortunately, for the phenol, the control and neutralized disinfectant wells were not statistically equivalent. In fact, there was a statistically significant difference between the mean LD of the biofilm subjected to the "neutralized phenol" wells compared to the controls ( $p$-value $<0.00005)$. Thus, the phenol in row A of the MBEC device did not appear to be adequately neutralized in the 8-lab study.

\subsection{Ruggedness testing}

\subsubsection{Controls}

Fig. 3 depicts the LDs of the control biofilms for both technicians across the 9 experimental conditions outlined in Table 2 . The tech-totech variability for the 4 SOP runs was estimated to be zero and was not statistically significant ( $p$-value $=0.751$ ). As the four factors were

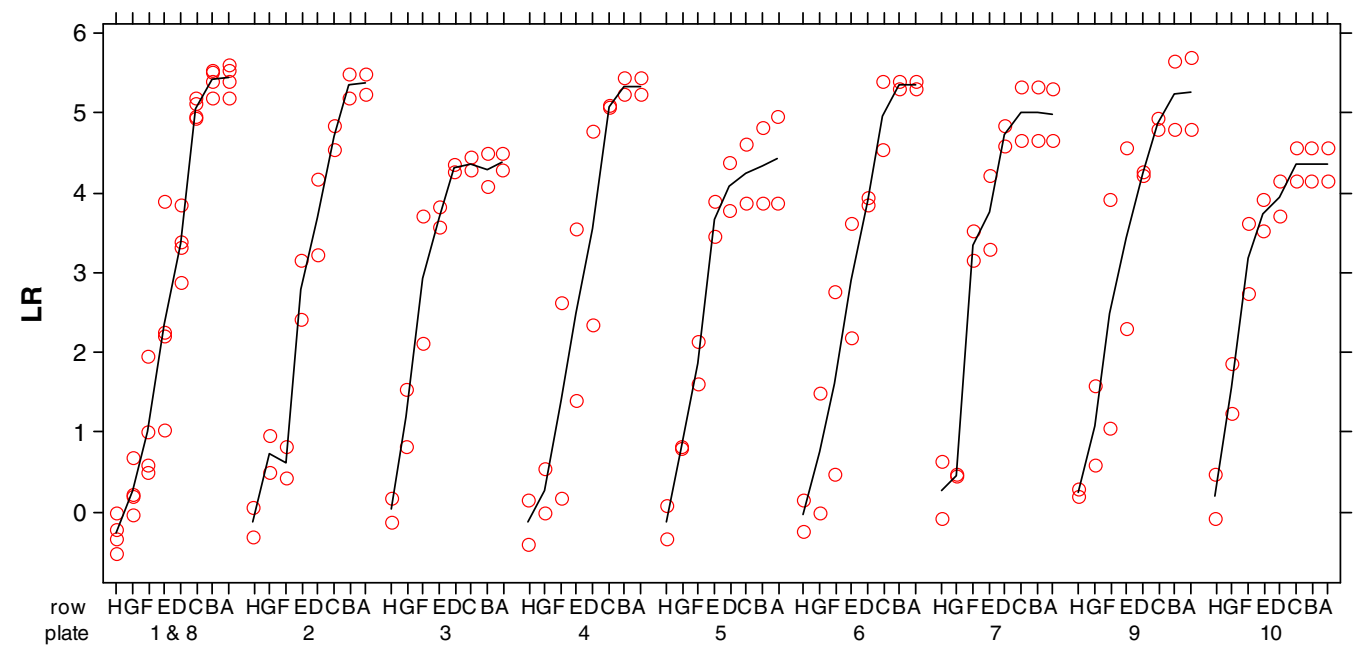

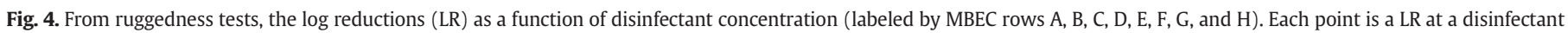
concentration for one of two technicians. The plate numbers refer to the sets of experimental conditions in Table 2. 
Table 5

Summary statistics for the regression equation fit to the log reductions (LR) from the ruggedness tests. Units for the factors are provided in Table 1 . The model depicted here contains all main effects and only statistically significant 2-way interactions.

\begin{tabular}{lrlc}
\hline Factors & Estimate & SE & $p$-value \\
\hline Intercept & 0.2157 & 0.9206 & 0.8151 \\
Inc-17 & -0.4570 & 0.1308 & 0.0036 \\
SonDur-30 & 0.0015 & 0.0152 & 0.9240 \\
Temp-22 & 0.0696 & 0.0654 & 0.3052 \\
Power-250 & 0.0003 & 0.0004 & 0.5389 \\
DisConc & 0.7866 & 0.0272 & $<0.00005$ \\
(Inc-17) $\times$ DisConc & 0.0832 & 0.0304 & 0.0071 \\
(Temp-22) $\times$ DisConc & -0.0305 & 0.0152 & 0.0467 \\
\hline
\end{tabular}

varied from the SOP values (according to Table 2), there were no significant two-way interaction effects on the control LDs ( $p$-value $\geq 0.2350$ ). The model fit to the mean control LD with only main effects is

$\mathrm{LD}=5.03+0.1111 *($ Inc -17$)-0.0042 *($ SonDur -30$)-0.1178 *($ Temp -22$)$ $+0.0004 *($ Power -250$)+0.3893 *($ BiofilmGrowth -5.87$)$.

The equation has been centered at the middle values of each of the factors. Hence, $5.03 \log _{10}\left(\mathrm{CFU} / \mathrm{mm}^{2}\right)$ is the predicted mean LD of the control biofilms when all of the operational factors are set to their SOP values, and when the mean $\mathrm{LD}$ of the five biofilm growth check pegs ("PC") is $5.87 \log _{10}\left(\mathrm{CFU} / \mathrm{mm}^{2}\right)$.

Table 4 summarizes the statistical analysis of the control biofilm LDs. None of the factors had a significant effect on the mean control LD ( $p$-value $\geq 0.0601$ ). The small coefficients for the factors in the equation for the mean control LD explicitly show the ruggedness of the MBEC assay to changes in the four factors. For example, the coefficient of -0.0042 for SonDur estimates that for every increase of sonication duration by $1 \mathrm{~min}$ (in the range 25-35 $\mathrm{min}$ ), the mean LD of the control wells will decrease on average by $0.004 \log _{10}\left(\mathrm{CFU} / \mathrm{mm}^{2}\right)$. Keep in mind that calculations based on this equation are valid only for values of the factors in the ranges outlined in Table 1.

\subsubsection{Treated data}

The LRs are given as a function of the 8 concentrations of disinfectant (in rows $\mathrm{A}-\mathrm{H}$ of the MBEC challenge plate) in Fig. 4. The LRs calculated from the 4 SOP runs (plates 1 and 8 per technician) are plotted in the left panel of Fig. 4. None of the two-way interactions amongst the 4 factors had a significant effect on the LRs ( $p$-value $\geq 0.3522$ ). None of the 3-way interactions with Disinfectant Concentration were significant either
( $p$-value $\geq 0.1795$ ). However, two-way interactions between Disinfectant Concentration and each of treatment temperature and incubation were significant ( $p$-value $\leq 0.0467)$. The model that included the significant two-way interactions and all of the main effects for the four factors is

$$
\begin{aligned}
\mathrm{LR}= & 0.2157-0.457 *(\text { Inc }-17)+0.0015 *(\text { SonDur }-30)-0.0696 *(\text { Temp }-22) \\
& +0.0003 *(\text { Power }-250)+0.7866 * \text { DisConc } \\
& -0.0305 *(\text { Temp }-22) * \text { DisConc }+0.0832 *(\text { Inc }-17) * \text { DisConc }
\end{aligned}
$$

This equation has been centered at the middle values of the factors. Thus, the constant 0.2157 is the predicted LR when DisConc $=0$ (corresponding a disinfectant concentration of $4.2 \mathrm{mg} / \mathrm{L}$ in row $\mathrm{H}$ ). When DisConc $=7$ (corresponding to $535 \mathrm{mg} / \mathrm{L}$ of disinfectant as in row $A$ ), the equation predicts a $L R=5.72$.

Table 5 gives a summary of the significance tests performed for each factor in the model. Just as for the control data, sonication duration $(p$-value $=0.9240)$ and sonication power $(p$-value $=0.5389)$ do not significantly affect the LR. The coefficient for DisConc is 0.7866 and is statistically significantly positive $(p$-value $<0.00005)$. Thus, when all of the factors are at their SOP values, as the disinfectant is increased up a single row of the MBEC device (e.g., going from row $\mathrm{E}$ to row $\mathrm{D}$, corresponding to DisConc increasing by 1 ), then it is predicted that the LR will increase by 0.7866 . This demonstrates that the MBEC assay was statistically significantly responsive to increasing disinfectant concentrations, which is a required attribute of an efficacy test. Again, we emphasize that the regression equation is statistically valid only within the ranges outlined in Table 1. It is not prudent to extrapolate beyond these values.

\subsection{Collaborative study}

The favorable ruggedness testing results indicated that the MBEC assay was ready for the next step in standardization, a collaborative study.

The untreated control LDs from the collaborative study are plotted in Fig. 5. Table 6 summarizes the means and variance components attributable to different sources of variability. The repeatability SD is given for each lab individually in order to identify labs with unusual variability. All of the repeatability and reproducibility SDs given in Table 6 are acceptable compared to results from historical data published by the Center for Biofilm Engineering (Table 1 in Parker and Hamilton, 2011). This indicates acceptable resemblance of the control data across multiple experiments in a single lab, and also across multiple laboratories.

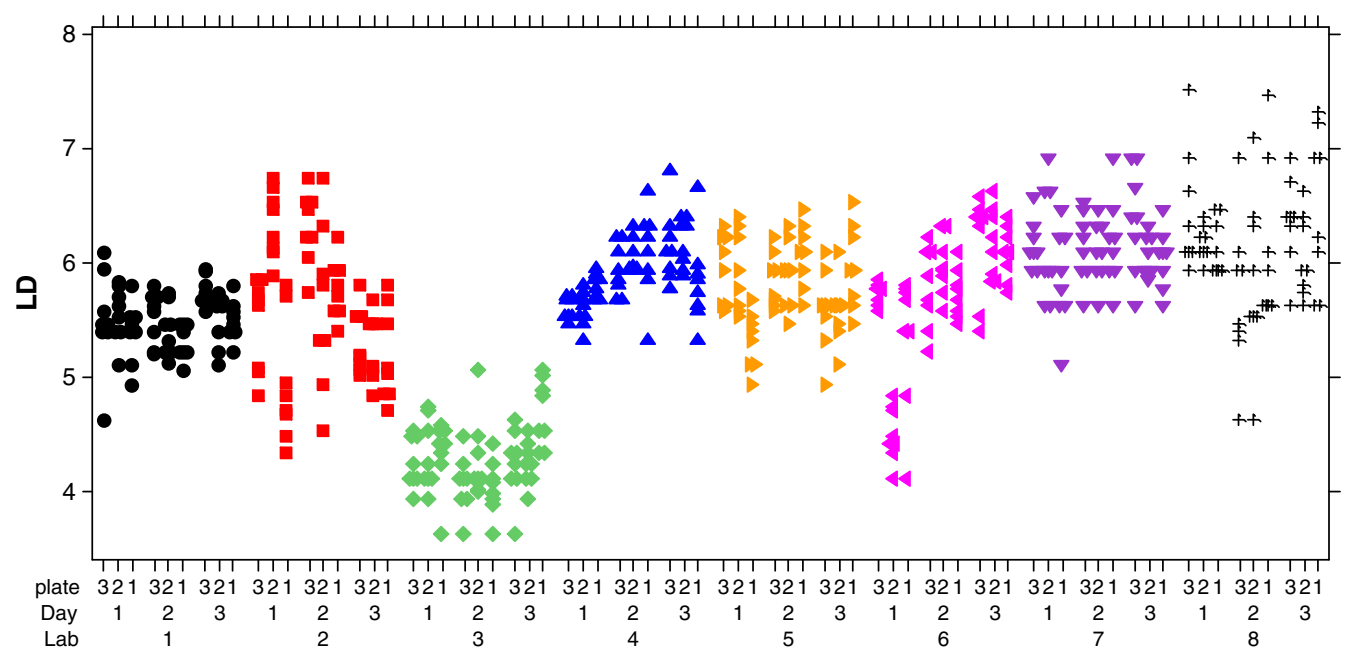

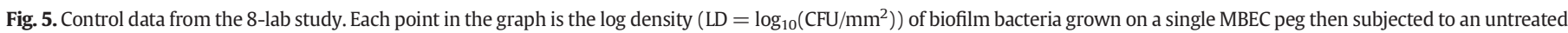
control well in an MBEC plate. Along the horizontal axis are listed the eight labs, the three experimental days within each lab, and the three plates within each experimental day. 
Table 6

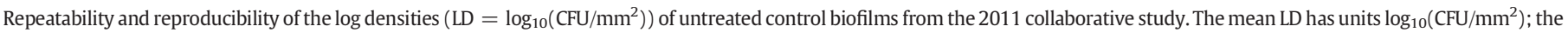
SDs are unitless.

\begin{tabular}{|c|c|c|c|c|c|c|c|c|}
\hline \multirow[t]{2}{*}{ Lab } & \multirow[t]{2}{*}{ No. exp } & \multirow[t]{2}{*}{ Mean LD } & \multicolumn{4}{|c|}{ Sources of variability } & \multirow[t]{2}{*}{ Repeatability SD } & \multirow[t]{2}{*}{ Reproducibility SD } \\
\hline & & & Within plate $\%$ & Among plate \% & Among exp day \% & Among lab \% & & \\
\hline 1 & 3 & 5.50 & $40 \%$ & $34 \%$ & $25 \%$ & & 0.1369 & \\
\hline 2 & 3 & 5.58 & $20 \%$ & $27 \%$ & $53 \%$ & & 0.4206 & \\
\hline 3 & 3 & 4.27 & $39 \%$ & $12 \%$ & $49 \%$ & & 0.1696 & \\
\hline 4 & 3 & 5.92 & $17 \%$ & $0 \%$ & $83 \%$ & & 0.2315 & \\
\hline 5 & 3 & 5.80 & $64 \%$ & $0 \%$ & $36 \%$ & & 0.1624 & \\
\hline 6 & 3 & 5.72 & $8 \%$ & $7 \%$ & $85 \%$ & & 0.5301 & \\
\hline 7 & 3 & 6.13 & $76 \%$ & $24 \%$ & $0 \%$ & & 0.1438 & \\
\hline 8 & 3 & 6.16 & $51 \%$ & $0 \%$ & $49 \%$ & & 0.2706 & \\
\hline All 8 & 24 & 5.48 & $4 \%$ & $11 \%$ & $9 \%$ & $76 \%$ & 0.3252 & 0.6669 \\
\hline
\end{tabular}

For each disinfectant type considered in the collaborative study, Figs. 6-8 present the LRs as a function of increasing disinfectant efficacy. Figs. 9-10 provide a graphical summary of the repeatability and reproducibility SDs respectively for each of the 24 disinfectant and concentration combinations.

The increasing LR curves in Figs. 6-8 suggest that, for each disinfectant, the MBEC assay is responsive to increasing disinfectant efficacy. The model fit to the LRs for each disinfectant provides a quantitative linear measure of the responsiveness of the method to increasing disinfectant efficacy. Table 7 shows that the MBEC assay is statistically significantly responsive to the increasing disinfectant efficacy for all three disinfectants.

\section{Discussion}

A laboratory method is considered ready for standardization if it has a successful publication record by different researchers in different labs, provides quantitative results, and uses typical equipment requiring typical laboratory expertise (Hamilton et al., 2013). The MBEC assay met these criteria with more than a decade of referenced use (Ceri et al., 1999). The next step in the standardization process for the MBEC assay was to conduct a ruggedness test with respect to important operational factors in the protocol.

Since biofilm bacteria are attached to a surface, the bacteria must be removed and disaggregated in order to provide unbiased estimates of the density of biofilm bacteria (Hamilton et al., 2009). Given the use of sonication for this purpose in the MBEC assay (and in many microbiological tests), it was imperative to assess the ruggedness of the MBEC assay with respect to factors that control sonication. Previous research has shown that sonication can affect antimicrobial efficacy measures such as the LR. Monsen et al. (2009) showed that increasing sonication duration reduced gram-negative bacterial biofilms, including $P$. aeruginosa. The effect of sonication is even more pronounced when used in conjunction with antimicrobials or at increased temperatures (Piyasena et al., 2003; Fux et al., 2004; Monsen et al., 2009). Piyasena et al. suggest that sonication kills by "thinning cell membranes," which could explain the results by Monsen et al. that gram-positive bacteria with thicker cell walls are less susceptible to sonication. Fux et al. suggest that synergistic kill occurs because sonication effectively disaggregates biofilms, thereby increasing the efficacy of an antimicrobial. Furthermore, and of great import for this study, Monsen et al. reported significant among-experiment and among-apparatus variability in their sonication experiments. The two sonication factors considered in the ruggedness tests for the MBEC were sonication duration and power. In addition, incubation time for growing the biofilms, and temperature during the disinfectant contact time with the biofilm, were also assessed. Based on the previous research, two-way interactions between the sonication factors and each of treatment temperature and disinfectant concentration were of particular interest.

Overall, the ruggedness testing showed that the results from the MBEC assay are not influenced by small changes of the operational factors. Interestingly, the mean control LDs were not significantly affected by any of the four factors (Table 4). When a disinfectant was applied, only incubation time and treatment temperature had statistically significant effects on the LRs, and these effects depended on the disinfectant concentration. Importantly, none of the two-way interactions among the four factors had a statistically significant effect on the LR (Table 5). Explicit equations (in Tables 4-5) quantify the simultaneous effect of

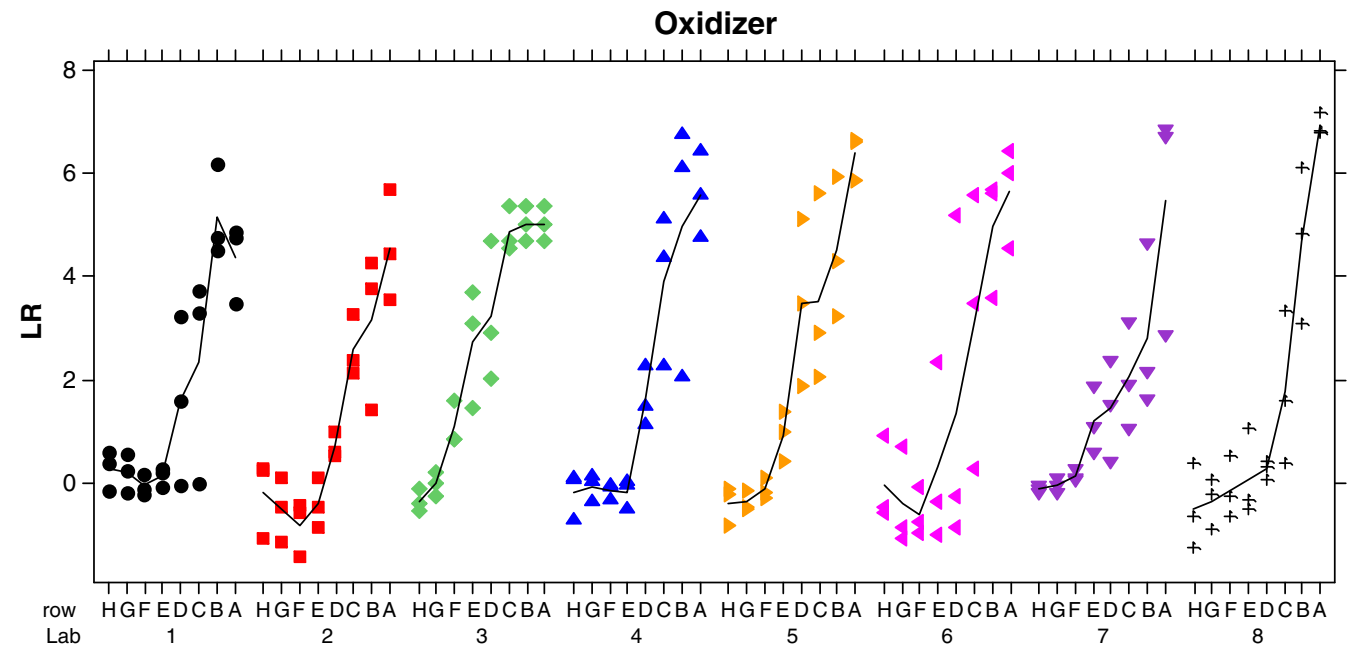

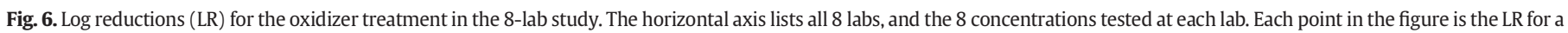
single concentration of disinfectant in a single experiment. 


\section{Phenol}

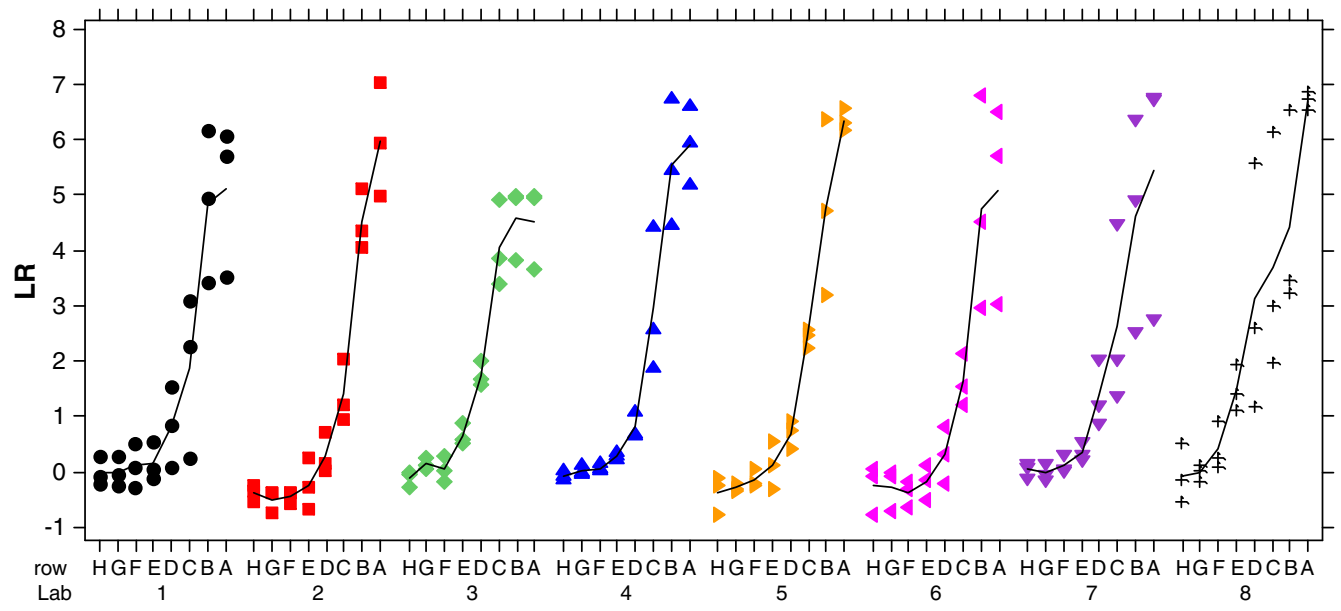

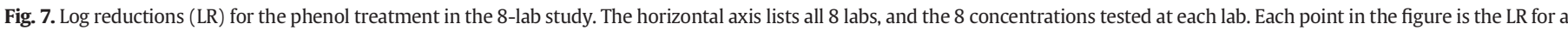
single concentration of disinfectant in a single experiment.

all 4 of these factors on the mean LD of untreated control biofilms grown in the MBEC; and also on the LR of the biofilm after application of multiple concentrations of a non-chlorine oxidizer. The small coefficients in these equations demonstrate precisely how rugged the MBEC results are to changes in these factors. For example, the coefficient of 0.0015 for SonDur (Table 5) predicts that as sonication duration increases from 25 to $35 \mathrm{~min}$, the LR will increase on average by 0.015 , provided that disinfectant concentration and the other factors stay constant. Similarly, as sonication power is increased by $100 \mathrm{~W}$ (in the range $130-480 \mathrm{~W}$ ), it is predicted that the LR will increase on average by 0.03 . Due to statistically significant interactions with disinfectant concentration, the effects of treatment temperature and incubation time do not have this same simple interpretation. Instead, the effects of these 2 factors depend on the disinfectant concentration.

Upon completion of the ruggedness tests, the estimates of MBEC variability were crucial since they were our first quantitative assessment of the repeatability of the MBEC across multiple experiments. These estimates are consistent with what was found with more confidence later in the 8-lab study, and so are not reported in this paper. One assessment of interest was that the technician-to-technician variability for the four ruggedness test SOP runs was estimated to be zero (i.e., between
$5.78 \times 10^{-12}$ and $\left.1.26 \times 10^{-11}\right)$ and not statistically significant for either the control LDs ( $p$-value $\geq 0.751$ ), or the LRs attained by the high concentrations of the oxidizer (in MBEC rows A-D, $p$-value $\geq 0.553$ ). For the less-efficacious treatments (in rows E-H), the technician-to-technician variance was estimated to be larger than 0 , but not statistically significant ( $p$-value $\geq 0.156$ ). These results were expected since the experimental design purposely sought to minimize technician-to-technician differences by requiring that the technicians independently conduct experiments side-by-side on the same day so that the effect of the factors could be studied with more statistical power. In general, a method with large technician-to-technician variability would not be suitable for standardization and would likely have a large reproducibility standard deviation.

The advantage of beginning the standardization process with ruggedness testing is that it can predict how well (or poorly) a method will perform across multiple labs while only requiring experiments from a single lab. For example, the initial MBEC protocol specified that incubation time could be set in the range 16-24 h (Table 1). Based on the ruggedness test results, the incubation time range in the standardized MBEC method was tightened to 16-18 h. Other than this change, the ruggedness test results indicated that the MBEC assay was well suited for the next step required for standardization, the collaborative

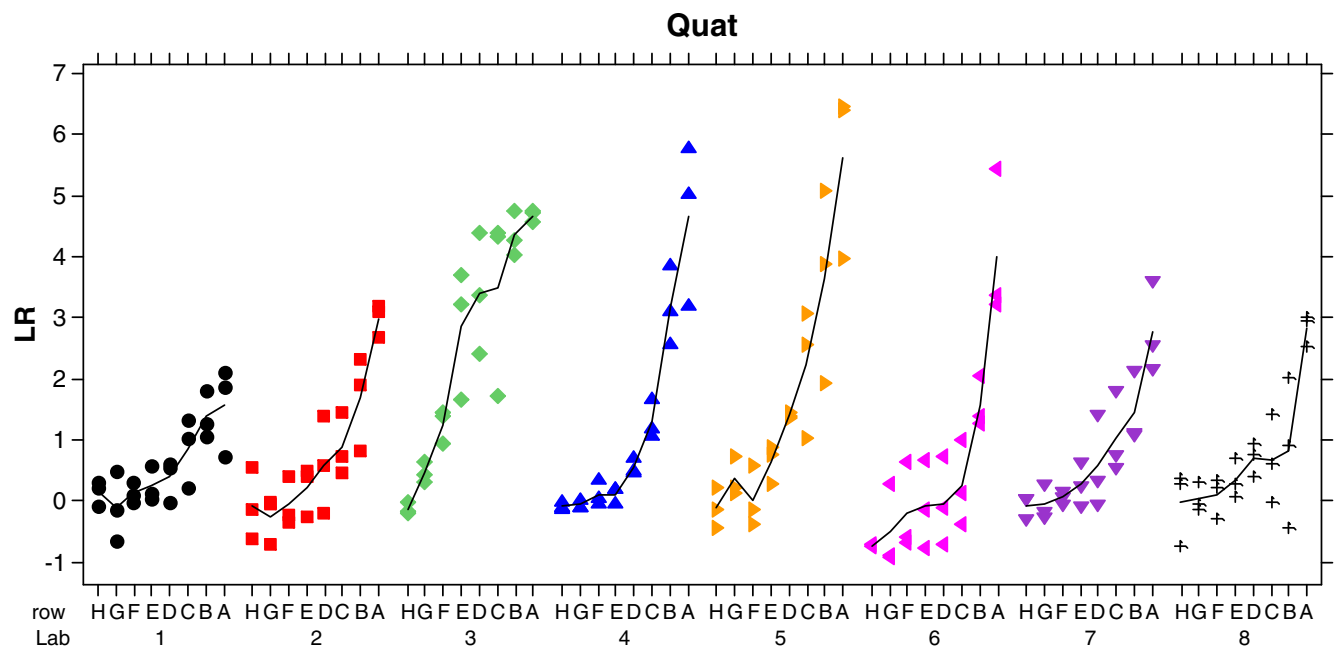

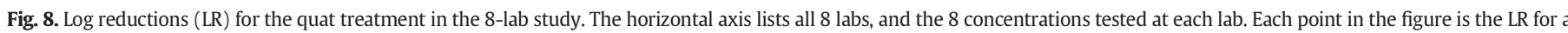
single concentration of disinfectant in a single experiment. 
study. The ruggedness test results also made it clear that in the multilaboratory study, in addition to incubation time, treatment temperature would need to be tightly monitored and controlled.

In preparation of the collaborative study, much effort was expended to identify a neutralizer that satisfactorily neutralized all 3 disinfectants at all 8 concentrations. As described earlier, while the oxidizer and quat were effectively neutralized (on the average) by $\mathrm{D} / \mathrm{E}$ broth during the collaborative study, the high concentration of the phenol (i.e., at least the $100 \%$ concentration) was not. This may explain why the $100 \%$ concentration of phenol attained the highest mean LR $(=5.64)$ in the 8-lab study. More generally, these results underscore that, compared to assays of planktonic bacteria, it is more challenging to neutralize the highly concentrated chemicals formulated to be effective against biofilms. One benefit of the MBEC is that disinfectant neutralization is monitored in every plate. This is an advantage over other disinfectant test methods, where neutralization tests are usually performed ahead of time in a separate experiment, and then neutralization is assumed to be adequate for all later experimental runs.

The collaborative study design for the MBEC assay satisfied ASTM's minimum requirements of 6 labs, 3 products, and 3 repeats of each product at each lab. As advocated by ASTM (E691), based on the multi-lab study data, we assessed the repeatability of the biofilm LRs across experiments, and the reproducibility across labs. In addition, since methods designed to test antimicrobials are based on an underlying biological system, we also assessed 3 other criteria that are not advocated by ASTM (E691): disinfectant neutralization, resemblance of the controls across labs, and responsiveness of the assay to increasing disinfectant efficacy (Hamilton et al., 2013).

The MBEC assay's performance in the collaborative study is depicted in Figs. 9-10. The frown-shaped quadratic relationship of the SDs as a function of disinfectant efficacy is to be expected for any disinfectant test method (Springthorpe and Sattar, 2005). This is because, for disinfectants with large LRs, most of the viable biofilm bacteria on the treated pegs are killed, which results in small variability of the treated LDs, so the observed variability for the LRs is close to the variability for the untreated control LDs. For low efficacy disinfectants with small LRs, the viable bacteria on the treated pegs resemble the viable bacteria on the untreated pegs, so, again, the observed variability for the LRs is close to the variability for the untreated control LDs. For moderately effective treatments, only some of the biofilm bacteria are killed, which results in a more variable LR. Since the three disinfectant types used in the collaborative study are considered representatives of classes of disinfectants, the actual concentrations of the disinfectants tested in

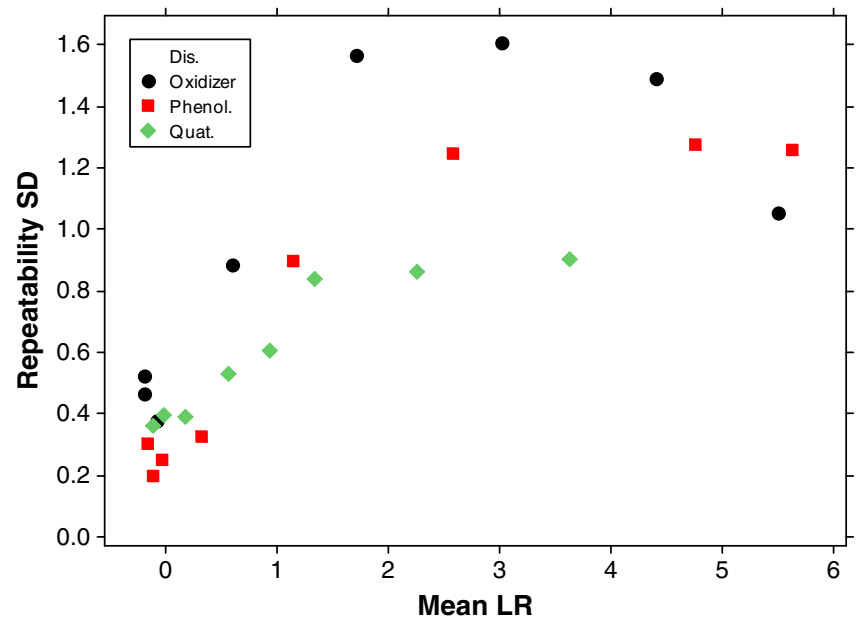

Fig. 9. For the collaborative study, the repeatability SDs calculated as a function of the mean log reduction (LR) for all 24 combinations of the 3 disinfectants and the 8 concentrations tested.

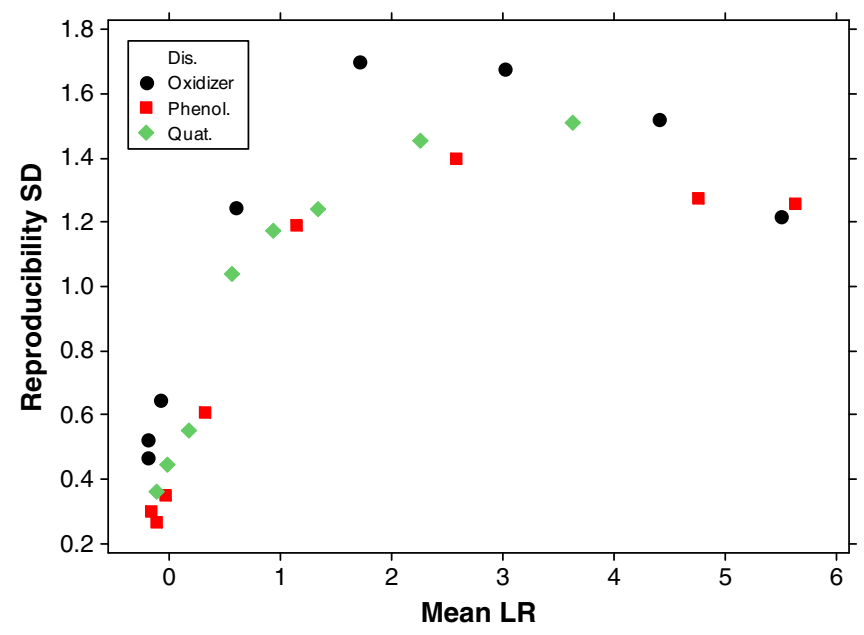

Fig. 10. For the collaborative study, the reproducibility SDs calculated as a function of the mean log reduction (LR) for all 24 combinations of the 3 disinfectants and the 8 concentrations tested.

the collaborative study are not as important as the mean LR achieved for each concentration of each disinfectant type.

Thus, the interested reader can, for example, predict the variability of a quat in a future MBEC study in a single lab by looking up the quat's mean LR in Fig. 9 and then reading off the attained repeatability SD for the quat in this study. Or one can predict the quat's variability in a future MBEC study across multiple labs by looking up the mean LR in Fig. 10 and then reading off the attained reproducibility SD for the quat in this study. Either of these scenarios could occur, for example, when performing tests for registration of a product with a regulatory agency.

From a regulator's point of view, the repeatability and reproducibility SDs reported in this paper can be used to craft performance standards for the MBEC method in order to determine the number of tests (i.e., independent plates), and the number of replicate wells in each plate, in order to achieve a target LR with a desired level of confidence (Parker et al., 2014). Based on another collaborative study (Tomasino et al., 2012), this approach was recently used to update the performance standard for the use-dilution method (UDM), the current method used by EPA to register products for hospital disinfectant claims (Tomasino et al., 2014). In order to have confidence that a performance standard for a method correctly passes effective products, while at the same time correctly failing ineffective ones, it is imperative to know the repeatability and reproducibility of the method.

\section{Conclusion}

In vitro studies remain a cornerstone for research, development, and registration of a variety of disinfectant and medical technologies. Understanding the performance of the underlying method in the controlled environment of the laboratory is a crucial step in order to provide convincing data that is repeatable across experiments, reproducible across labs, responsive to varying treatment efficacies, and rugged to changes in factors that may have been changed from the protocol. Extensive testing is required to assess these attributes for any method. Based on

Table 7

From the 2011 collaborative study, responsiveness of the MBEC to increasing concentrations of disinfectant. The trend is interpreted as the mean increase in the log reduction (LR) as the concentration of disinfectant increases two-fold (as occurs when moving up one row in the MBEC plate).

\begin{tabular}{llll}
\hline Disinfectant & Trend & SE & $p$-value \\
\hline Non-chlorine oxidizer & 0.87 & 0.0379 & $<0.00005$ \\
Phenol & 0.87 & 0.0376 & $<0.00005$ \\
Quat & 0.50 & 0.0275 & $<0.00005$ \\
\hline
\end{tabular}


ruggedness tests and the 8-lab study performed in 2011, these attributes for the MBEC assay were assessed and found to be acceptable by ASTM's subcommittee on antimicrobial agents (E35.11). Thus, the MBEC method is a low shear, high throughput, batch system that is rugged, responsive, repeatable and reproducible; and complements the suite of reactor systems available for biofilm growth and disinfection.

\section{Acknowledgments}

The authors are grateful to the 8 labs that volunteered their time and resources to participate in the inter-laboratory study. We also would like to thank ASTM for providing the technical expertise in the experimental design and for supporting the statistical analysis of the multilab study. Innovotech conducted the ruggedness tests, supported the statistical analysis of the ruggedness tests, and provided the treatment chemicals to the labs in the collaborative study. Innovotech also partially supported the writing of this manuscript.

\section{References}

ASTM research report RR:E35-1006, 2011. Interlaboratory Study for ASTM Method E2799-11 Standard Test Method for Testing Disinfectant Efficacy against Pseudomonas aeruginosa Biofilm Using the MBEC Assay. ASTM International, West Conshohocken, PA.

ASTM standard E1054, 2013. Standard Test Methods for Evaluation of Inactivators of Antimicrobial Agents. ASTM International, West Conshohocken, PA.

ASTM standard E2196, 2012. Standard Test Method for Quantification of Pseudomonas aeruginosa Biofilm Grown with Medium Shear and Continuous Flow Using Rotating Disk Reactor. ASTM International, West Conshohocken, PA.

ASTM standard E2562, 2012. Standard Test Method for Quantification of Pseudomonas aeruginosa Biofilm Grown with High Shear and Continuous Flow using CDC Biofilm Reactor. ASTM International, West Conshohocken, PA.

ASTM standard E2647, 2013. Standard Test Method for Quantification of Pseudomonas aeruginosa Biofilm Grown Using Drip Flow Biofilm Reactor with Low Shear and Continuous Flow. ASTM International, West Conshohocken, PA.

ASTM standard E2799, 2012. Standard Test Method for Testing Disinfectant Efficacy against Pseudomonas aeruginosa Biofilm using the MBEC Assay. ASTM International, West Conshohocken, PA.

ASTM standard E2935, 2013. Standard Practice for Conducting Equivalence Testing in Laboratory Applications. ASTM International, West Conshohocken, PA.

ASTM standard E691, 2013. Standard Practice for Conducting an Interlaboratory Study to Determine the Precision of a Test Method. ASTM International, West Conshohocken, PA.

Behnke, S., Parker, A., Woodall, D., Camper, A., 2011. Comparing the chlorine disinfection of detached biofilm clusters with sessile biofilms and planktonic cells in single and dual species cultures. Appl. Environ. Microbiol. 77 (20), 7176-7184.

Buckingham-Meyer, K., Goeres, D., Hamilton, M., 2007. Comparative evaluation of biofilm disinfectant efficacy tests. J. Microbiol. Methods 70, 236-244.

Ceri, H., Olson, M.E., Stremick, C., Read, R.R., Morck, D., Buret, A., 1999. The Calgary Biofilm Device: new technology for rapid determination of antibiotic susceptibilities of bacterial biofilms. J. Clin. Microbiol. 37, 1771-1776.
Davies, D., 2003. Understanding biofilm resistance to antibacterial agents. Nat. Rev. Drug Discov. 2, 114-122.

Donlan, R.M., Costerton, J.W., 2002. Biofilms: survival mechanisms of clinically relevant microorganisms. Clin. Microbiol. Rev. 15, 167-193.

Fux, C.A., Wilson, S., Stoodley, P., 2004. Detachment characteristics and oxacillin resistance of Staphylococcus aureus biofilm emboli in an in vitro catheter infection model. J. Bacteriol. 186, 4486-4491.

Goeres, D., Loetterle, L., Hamilton, M., Murga, R., Kirby, D., Donlan, R., 2005. Statistical assessment of a laboratory method for growing biofilms. Microbiology 151, 757-762.

Goeres, D.M., Hamilton, M.A., Beck, N.A., Buckingham-Meyer, K., Hilyard, J.D. Loetterle, L.R., Lorenz, L.A., Walker, D.K., Stewart, P.S., 2009. A method for growing a biofilm under low shear at the air-liquid interface using the drip flow biofilm reactor. Nat. Protoc. 4 (5), 783-788.

Hamilton, M.A., Buckingham-Meyer, K., Goeres, D.M., 2009. Checking the validity of the harvesting and disaggregating steps in laboratory tests of surface disinfectants. JAOAC 92, 1755-1762.

Hamilton, M., Hamilton, G., Goeres, D., Parker, A., 2013. Guidelines for the statistica analysis of a collaborative study of a laboratory method for testing disinfectant product performance. JAOAC 96 (5)

Harrison, J., Stremick, C., Turner, R., Allan, N., Olson, M., Ceri, H., 2010. Microtiter susceptibility testing of microbes growing on peg lids: a miniaturized biofilm model for high-throughput screening. Nat. Protoc. 5, 1236-1254.

Monsen, T., Lövgren, E., Widerström, M., Wallinder, L., 2009. In vitro effect of ultrasound on bacteria and suggested protocol for sonication and diagnosis of prosthetic infections. J. Clin. Microbiol. 47 (8), 2496-2501.

Nelson, M., LaBudde, R., Tomasin, S., Pines, R., 2013. Comparison of 3M PetrifilmTM aerobic count plates to standard plating methodology for use with AOAC antimicrobial efficacy methods $955.14,955.15,964.02$, and 966.04 as an alternative enumeration procedure: collaborative study. JAOAC 96 (4).

Parker, A., Hamilton, M., 2011. Assessing resemblance, repeatability and reproducibility for quantitative methods. Center for Biofilm, Engineering, KSA-SM-10 (http://www. biofilm.montana.edu/content/ksa-sm-10).

Parker, A., Hamilton, M., 2013. Using R to assess resemblance, repeatability, and reproduc ibility for quantitative and semi-quantitative disinfectant methods. Center for Biofilm, Engineering, KSA-SM-13 (http://www.biofilm.montana.edu/content/ksa-sm-13).

Parker, A., Tomasino, S., Hamilton, M.A., 2014. Statistical model for assessing performance standards for quantitative and semi-quantitative disinfectant test methods. JAOAC 97 (1), 58-67.

Pinheiro, J., Bates, D., 2000. Mixed Effects Models in S and S-PLUS. Springer.

Pinheiro, J., Bates, D., DebRoy, S., Sarkar, D., R Development Core Team, 2012. nlme: linear and nonlinear mixed effects models. R package version 3.1-104.

Piyasena, P., Mohareb, E., McKellar, R.C., 2003. Inactivation of microbes using ultrasound: a review. Int. J. Food Microbiol. 87, 207-216.

R Core Team, 2012. R: A Language and Environment for Statistical Computing. R Foundation for Statistical Computing, Vienna, Austria 3-900051-07-0 (URL http://www.R-project. org/).

Richter, S.J., Richter, C., 2002. A method for determining equivalence in industrial applications. Qual. Eng. 14 (3), 375-380.

Springthorpe, S., Sattar, S., 2005. Carrier tests to assess microbicidal activities of chemical disinfectants for use on medical devices and environmental surfaces. JAOAC 88 (1), 182-201.

Tomasino, S., Parker, A., Hamilton, M., Hamilton, G., 2012. Performance of the AOAC use-dilution method with targeted modifications: collaborative study. JAOAC 95 (6), 1618-1628.

Tomasino, S., Parker, A., Hamilton, M., 2014. Use of statistical modeling to reassess the performance standard for the AOAC use-dilution methods (955.15 and 964.02). JAOAC 97 (1), 68-77. 